\title{
Histology and ultrastructure of the uterus of African giant rat (Cricetomys Gambianus, Waterhouse) during oestrous cycle
}

\author{
A.K. Akinloye ${ }^{1}$, B.O. Oke ${ }^{2}$ \\ ${ }^{1}$ Department of Veterinary Anatomy, Federal University of Agriculture, Abeokuta, Nigeria \\ ${ }^{2}$ Department of Veterinary Anatomy, University of Ibadan, Ibadan, Nigeria
}

[Received 22 February 2014; Accepted 8 April 2014]

\begin{abstract}
This study describes histology and ultrastructure of uterus in the African giant rat during oestrous cycle. Endometrial histology displayed glandular mucosa consisting of lamina epithelialis and lamina propria mucosae. Its epithelium varied between simple and pseudostratified columnar. The myometrium consisted of inner circular and outer longitudinal smooth muscles with medium sized arteries and veins in-between. The perimetrium contained simple squamous epithelium. Endometrial ultrastructures were variable during oestrous cycle. At mid oestrus, hemidesmosomes anchored undulating basement membrane of the mucosal epithelium. Preponderance spherical mitochondria, lipofuscin granules concentration, flocculent homogenous materials and indented nuclei were displayed. At mid metoestrus, late metoestrus/early dioestrus and mid dioestrus, the base of the mucosal columnar epithelium lain on relatively straight basement membrane and their cytoplasmic ultrastructure displayed variation to mid oestrus. Epithelial apex showed intermediate filament, microvilli and junctional complexes. The uterine glands occurred in variable numbers and sizes during oestrous cycle and shared similar ultrastructure. Mid dioestrus showed cell ultrastructure of uterine glands having apical accumulation of secretory vesicles. Some actively secreting uterine glands were lined by simple ciliated columnar epithelium mingled with pseudostratified epithelium. The findings of the study indicate that giant rat endometrial ultrastructure varies during oestrous cycle and glandular secretion is merocrine. (Folia Morphol 2015; 74, 3: 311-317)
\end{abstract}

Key words: uterus, oestrous, giant rat, ultrastructure, histology

\section{INTRODUCTION}

African giant rat (Cricetomys gambianus, Waterhouse) also known as the African Pouched rat, is a nocturnal pouched rat native to Africa, and an invasive alien species in Florida. The animals have been studied for use in detecting tuberculosis and in sniffing out land mines [12]. Besides that, Cricetomys rodents are traditionally hunted as food in most parts of Africa providing cheap source of protein $[8,10]$. Therefore, there is a renewed effort to characterise various aspects of their reproductive biology in order to improve their breeding potential.

The walls of the uterus consist of a luminal mucous membrane lining endometrium, an intermediate smooth muscle layer myometrium, and an external serous envelope, the peritoneum perimetrium. The endometrium is composed of two zones that differ in structure 
and function [5, 6]. Akinloye and Oke [2] had characterised the uterus of the African giant rat as a duplex uterus having two separate uterine horns and two cervices while Madekurozwa et al. [9] had investigated the immunolocalisation of the progesterone and oestrogen receptors in the uterine horns of the African giant rat during the oestrous cycle. Therefore, the present study was undertaken to investigate the ultrastructure of the uterus during oestrous cycle in order to complement and provide some insight into the previous studies.

\section{MATERIALS AND METHODS}

The Ethics Committee of the University of Ibadan, Nigeria, approved this study. Twenty-one sexually matured female African giant rats (Cricetomys gambianus), aged 26-28 weeks and weighing 800-950 g, were used in the present study. The animals were housed in temperature-controlled rooms, with a light regime of 12-h light and 12-h dark. Food and water were provided ad libitum. Vaginal smears were taken daily at between 08:00 am and 09:00 am to determine the stages of the oestrous cycle as described by Oke and Oke [11]. Only giant rats that exhibited at least two consecutive 5-day oestrous cycles were used in the study. Vaginal smears were taken prior to euthanasia to determine the stage of the oestrous cycle. Seven groups, each containing 3 animals, at different stages of oestrous cycle were identified as: mid pro-oestrus, late pro-oestrus/early oestrus and mid oestrus groups as well as mid metoestrus, late metoestrus/early dioestrus, mid dioestrus and late dioestrus/ early pro-oestrus groups. The animals were euthanased with an overdose of sodium pentobarbitone, dissected and thereafter, uterine samples were collected and processed for light microscopy as described by Akinloye et al. [1]. For transmission electron microscopy, the uterine tissues obtained from all the rats were immersion-fixed in $2.5 \%$ glutaraldehyde in $0.1 \mathrm{M}$ cacodylate buffer. Thereafter, the tissue samples were post-fixed in osmium tetroxide, dehydrated and embedded in epoxy resin. Ultrathin sections were stained with lead citrate and uranyl acetate. The samples were viewed with a Philips CMIO transmission electron microscope.

\section{RESULTS}

\section{Histology}

Figure 1 shows the basic histological architecture of the uterus in all stages of oestrous cycle. The wall of the uterus consisted of tunica mucosa (endometrium), tunica muscularis, (myometrium) and tunica

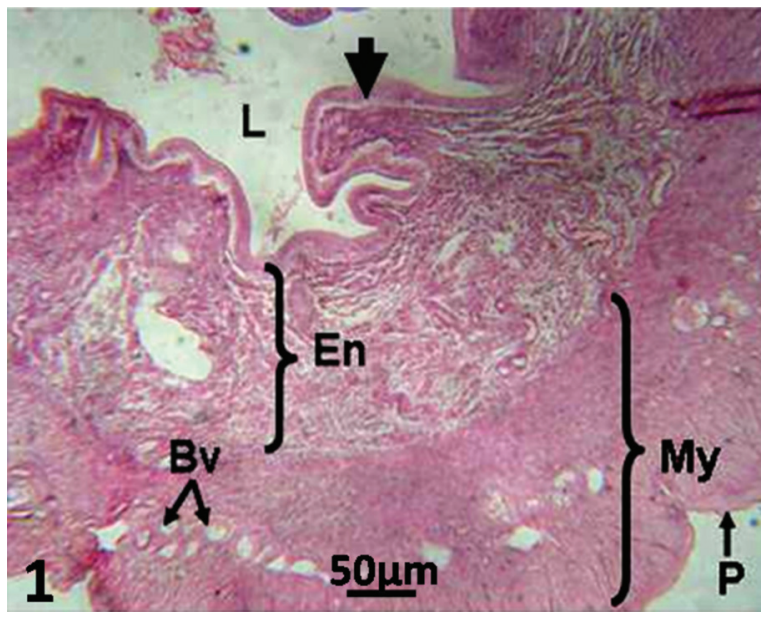

Figure 1. The uterus in the adult female African giant rat at mid metoestrus. Notice the wide endometrium (En) with its mucosal surface facing the uterine lumen $(\mathrm{L})$ and thrown into longitudinal folds (arrow). Also observe the relatively thick myometrium (My) interspersed with blood vessels (Bv) and surrounded by serosa, the perimetrium $(\mathrm{P})$; haematoxylin and eosin.

serosa (perimetrium). At mid pro-oestrus as well as in other stages of the oestrous cycle, the endometrium was observed to be a glandular mucosa consisting of lamina epithelialis and lamina propria mucosae. Its epithelial lining varied between simple columnar and pseudostratified columnar epithelia that formed low longitudinal folds. No sloughing of the epithelium was found in all the stages of the oestrous cycle. The lamina propria consisted of loose connective tissues, medium and small blood vessels as well as tubular glands. The tubular glands were observed to extend from the endometrium into the thick lamina propria and displayed little branching and coiling (Fig. 2). The myometrium consisted of inner circular and outer longitudinal smooth muscles with medium sized arteries and veins in-between. The perimetrium contained simple squamous epithelium.

\section{Ultrastructure}

Endometrial ultrastructures were variable at different stages of oestrous cycle. At mid oestrus, the basal part of the mucosal epithelium revealed undulating basement membrane anchored by hemidesmosomes. Preponderance of spherical mitochondria with lamellar cristae as well as concentration of lipofuscin granules, flocculent homogenous materials and indented nuclei were displayed (Fig. 3). At the apical part of the epithelium, short microvilli and typical junctional complexes were observed. Profiles of Golgi 


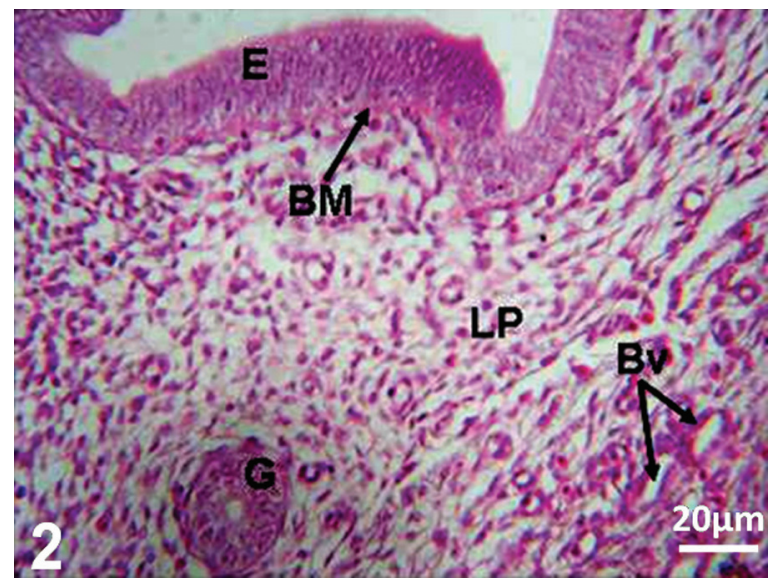

Figure 2. The endometrium in the adult female African giant rat uterus at mid pro-oestrus. Notice the pseudostratified/simple columnar epithelium (E) of the endometrial mucosa resting on relatively straight basement membrane (BM). Observe that the extensive lamina propria (LP) bears the glands $(\mathrm{G})$ and numerous blood vessels $(\mathrm{Bv})$; haematoxylin and eosin.

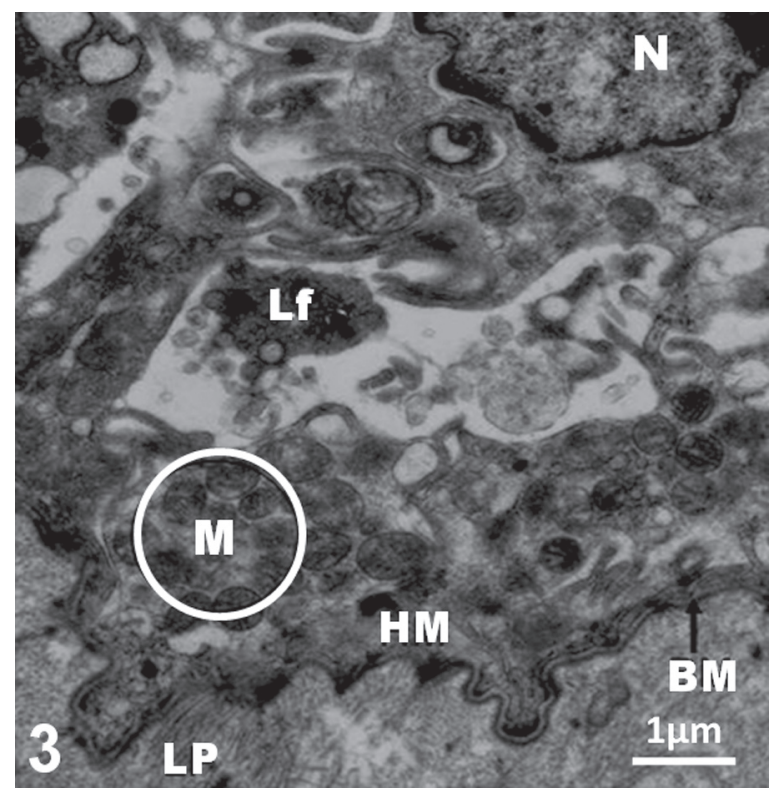

Figure 3. Basal part of uterine epithelium at mid oestrus. Observe the nucleus $(N)$, numerous mitochondria $(M)$ and hemidesmosomes (HM) at the basement membrane (BM). Notice the concentration of lipofuscin (Lf) granules. Below the epithelium is the lamina propria (LP).

stacks, numerous electron dense and electron lucid vesicles as well as rough endoplasmic reticulum, free ribosomes and even distribution of mitochondria were identified (Figs. 4, 5). At mid metoestrus, late meotestrus/early dioestrus and mid dioestrus, the basal part of the mucosal columnar epithelium laid on relatively straight basement membrane and their

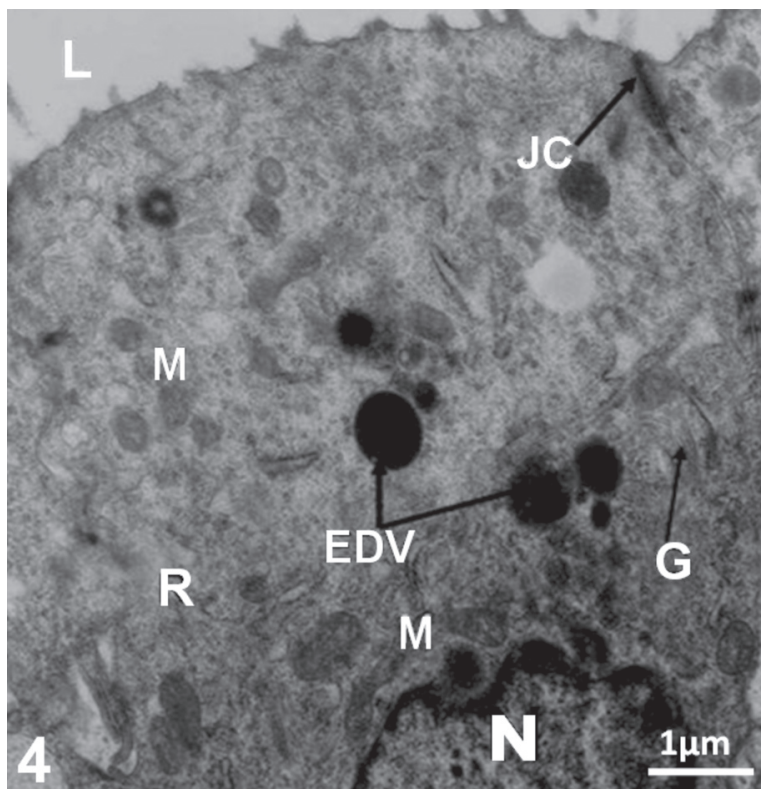

Figure 4. Apical part of endometrium of uterus at mid oestrus. Notice the junctional complex (JC) at the apical surface, lumen (L), numerous mitochondria $(\mathrm{M})$, variably shaped electron dense vesicles (EDV) and profiles of Golgi complex (G) as well as nucleus (N) and free ribosomes $(\mathrm{R})$.

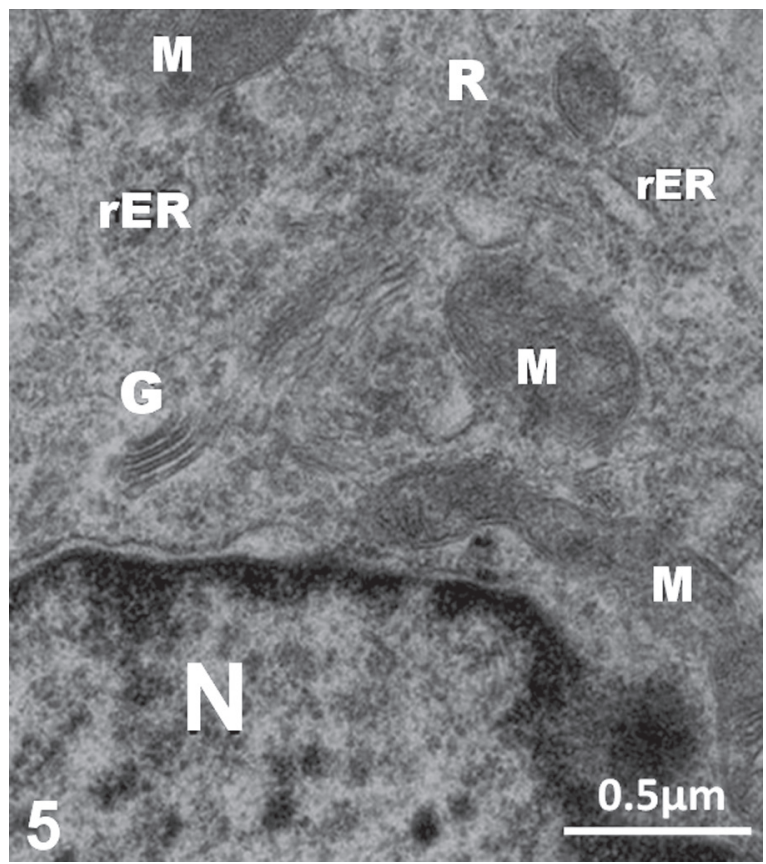

Figure 5. Higher magnification of a portion of Figure 4. Notice the variably shaped mitochondria (M), rough endoplasmic reticulum (rER), profiles of Golgi apparatus $(G)$, abundant free ribosomes $(R)$ and nucleus (N).

cytoplasmic ultrastructure showed variation to mid oestrus (Fig. 6). The luminal surface showed microvilli of variable lengths and glycocalyx. Condensation of 


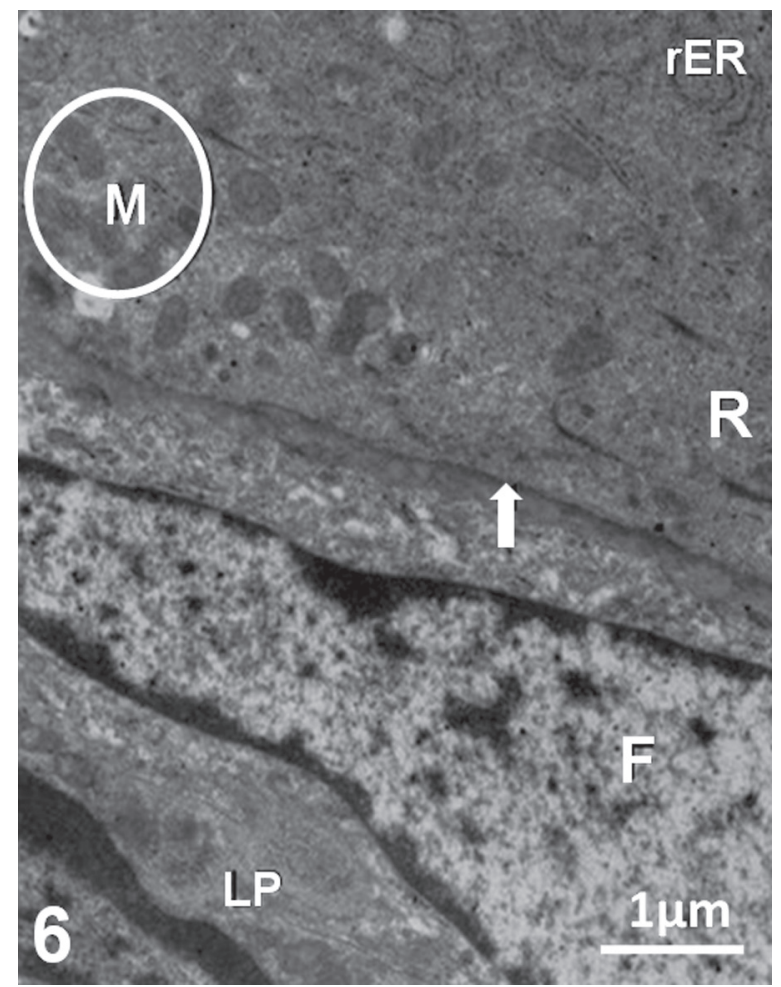

Figure 6. Basal part of endometrium of uterus at mid metoestrus. Notice the relatively straight basement membrane (arrow), accumulation of mitochondria (M), rough endoplasmic reticulum ( $\mathrm{rER}$ ) and ribosomes $(\mathrm{R})$. Observe the fibrocytes $(\mathrm{F})$ in the lamina propria $(\mathrm{LP})$.

intermediate filaments, electron dense and electron lucid vesicles was distinct at the apical region (Fig. 7). The ciliated columnar epithelium of the endometrium observed in late dioestrus/early prooestrus rested on slightly undulating basement membrane. The apical surface displayed numerous long microvilli and electron dense materials. Cytoplasmic organelles occurred in moderate numbers. Mitochondria concentrated at the basal region and were variably shaped. The lamina propria was highly cellular (Fig. 8).

Although the uterine glands occurred in variable numbers and sizes at different stages of the oestrous cycle, they partly shared similar ultrastructure. Mid dioestrus displayed well-developed uterine glands in the lamina propria. Some actively secreting uterine glands were lined with ciliated simple columnar, pseudostratified and stratified epithelia. The main difference between the mucosal and glandular epithelia was the occurrence of abundant ciliated cells in the glandular epithelium (Fig. 9). Uterine glands were surrounded by distinct basal lamina. Cellular ultrastructure did not differ significantly from that of surface epithelial cells (Fig. 10) but mitochondria concentra-

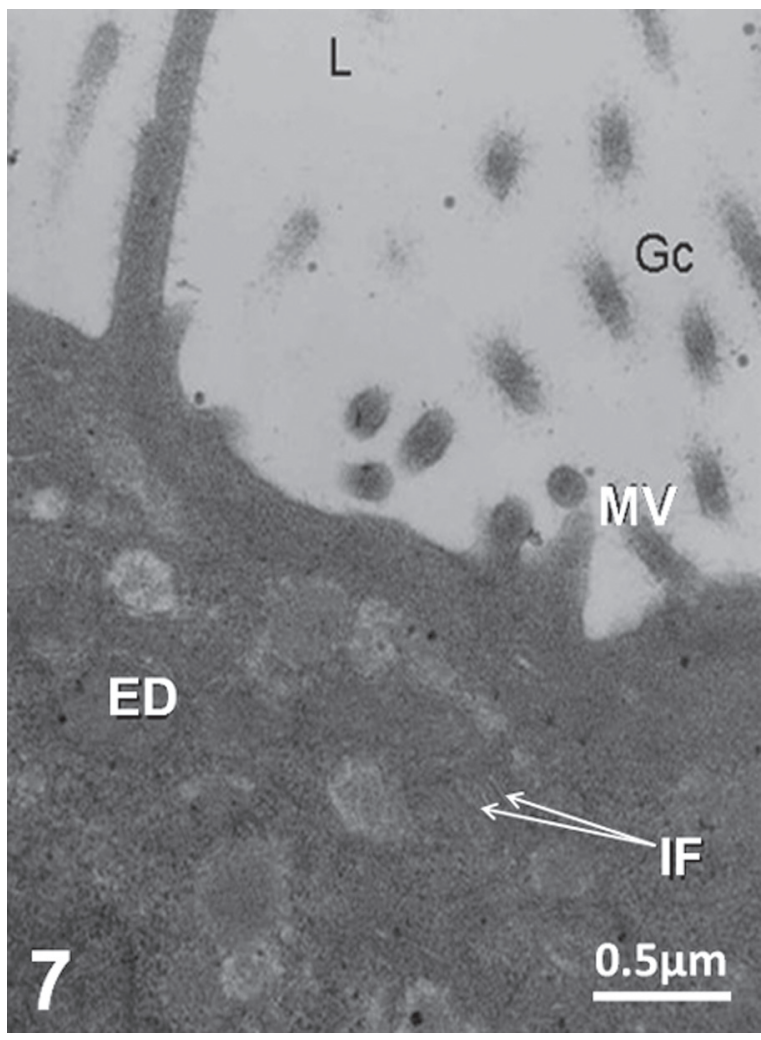

Figure 7. Apical part of the endometrium of uterus at mid metoestrus. Notice the microvilli (MV) and glycocalyx (Gc) on the luminal surface (L). Observe condensed intermediate filament (IF) and electron dense (ED) materials.

ted at the basal region and lateral in-folding of cell membranes were much greater and wide-spread in the glandular than in the mucosal epithelial cells (Fig. 11). The apical surface had accumulation of secretory vesicles and evidence of fusion of some vesicles with the cell membrane. The apex of the cells often bulged into the glandular lumen that was sparsely filled with glandular secretion and adjacent cells were connected via apical junctional complexes (Figs. 12, 13).

\section{DISCUSSION}

This study has shown that considerable parallel exists in histology of the uterus of the female African giant rat and other species of animals, especially the laboratory rat. The cyclic histological features of the various regions of the uterus observed at different stages of the oestrous cycle in the present study correlate with observations earlier reported in other animal species $[5,6,9,13]$. Results from the present study revealed that the uterine surface epithelia and the uterine glands share the same cyclic ultrastructural features and slight similarities exist among 


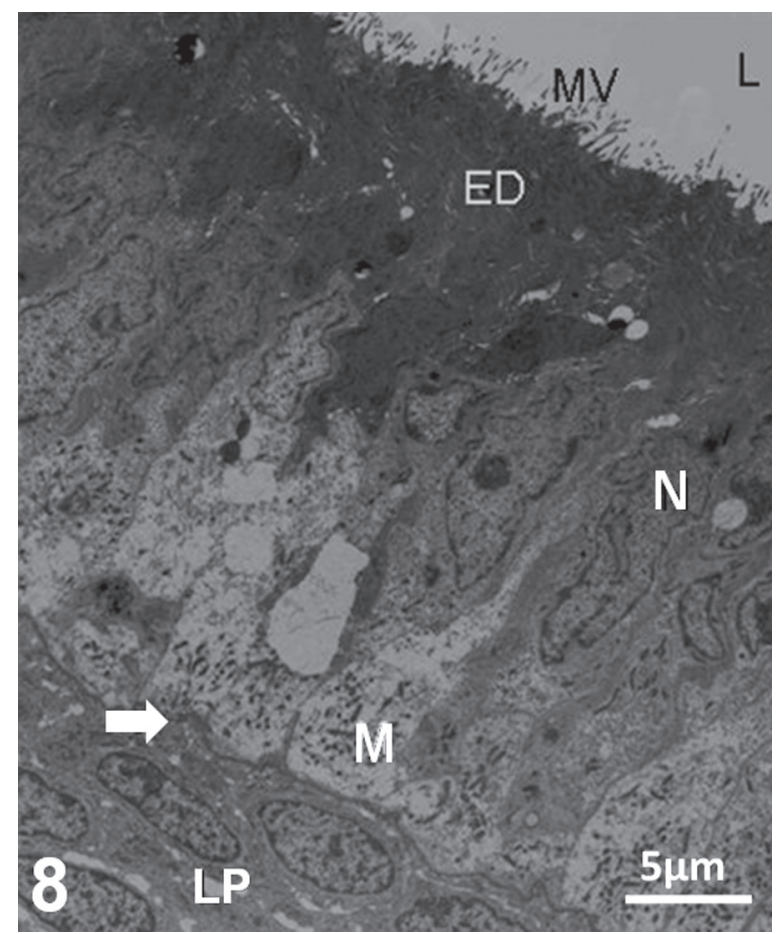

Figure 8. Uterine endometrium at late dioestrus/early pro-oestrus. Notice long microvilli (MV) projecting into the lumen (L), nucleus (N), condensed electron dense (ED) materials at the apical part and accumulation of mitochondria (M) at the basal part close to the lamina propria (LP). Observe the undulating basement membrane (arrow).

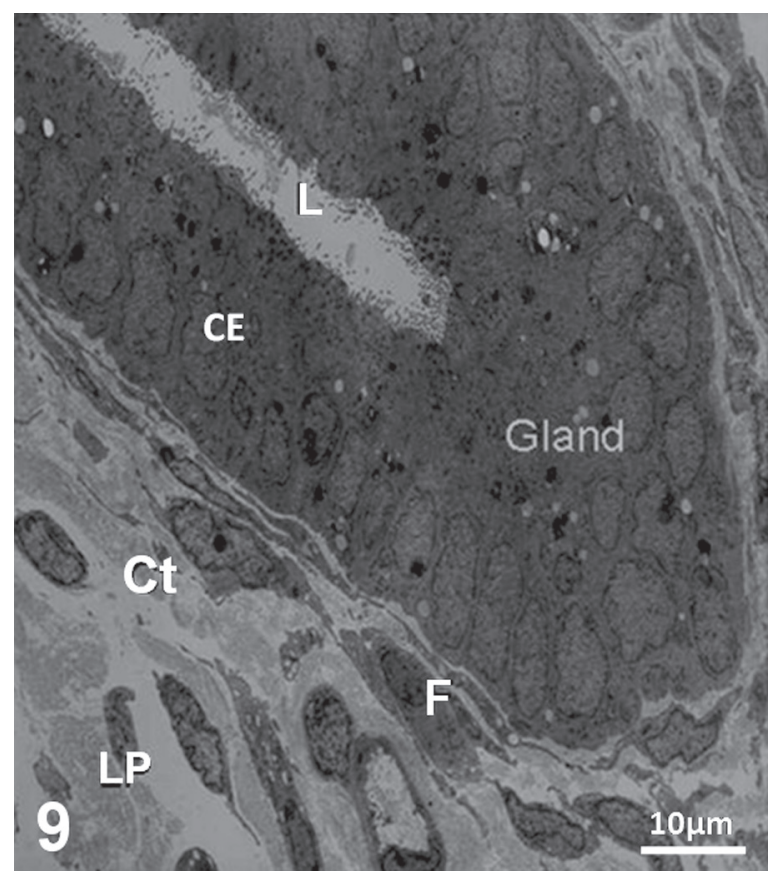

Figure 9. Uterine gland in the sub-mucosa of uterus at mid dioestrus. Notice the columnar epithelium (CE) of the gland in the lamina propria (LP) surrounded by connective tissue $(\mathrm{Ct})$ and fibrocytes (F). Observe scanty secretion in the glandular lumen (L).

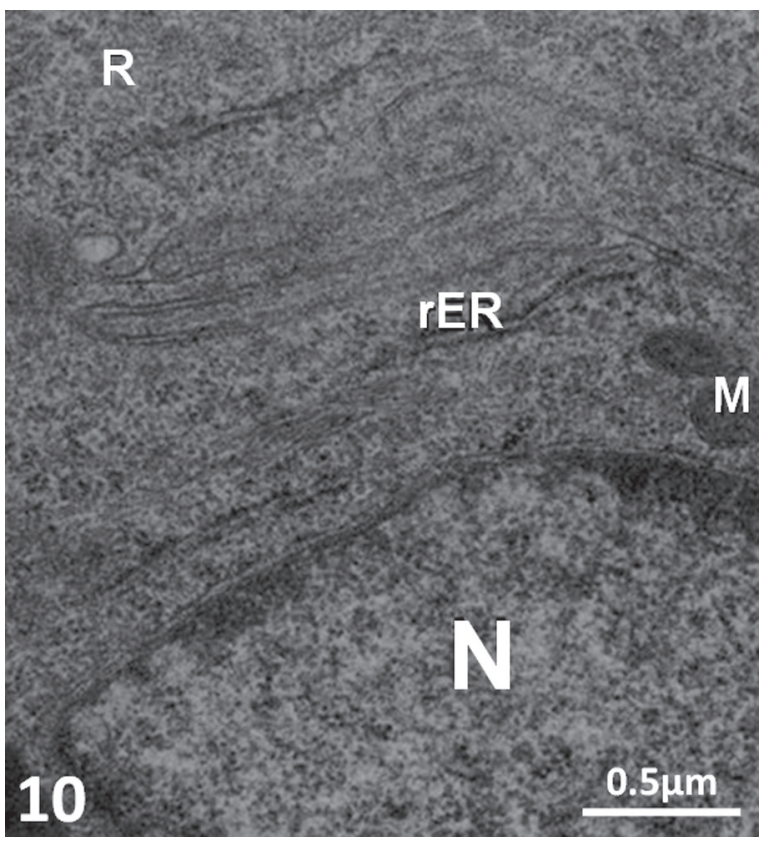

Figure 10. Cell of secretory gland in endometrial sub-mucosa at mid dioestrus. Notice the active nucleus $(\mathrm{N})$, profiles of rough endoplasmic reticulum ( $\mathrm{rER})$, dense free ribosomes $(\mathrm{R})$ and mitochondria (M).

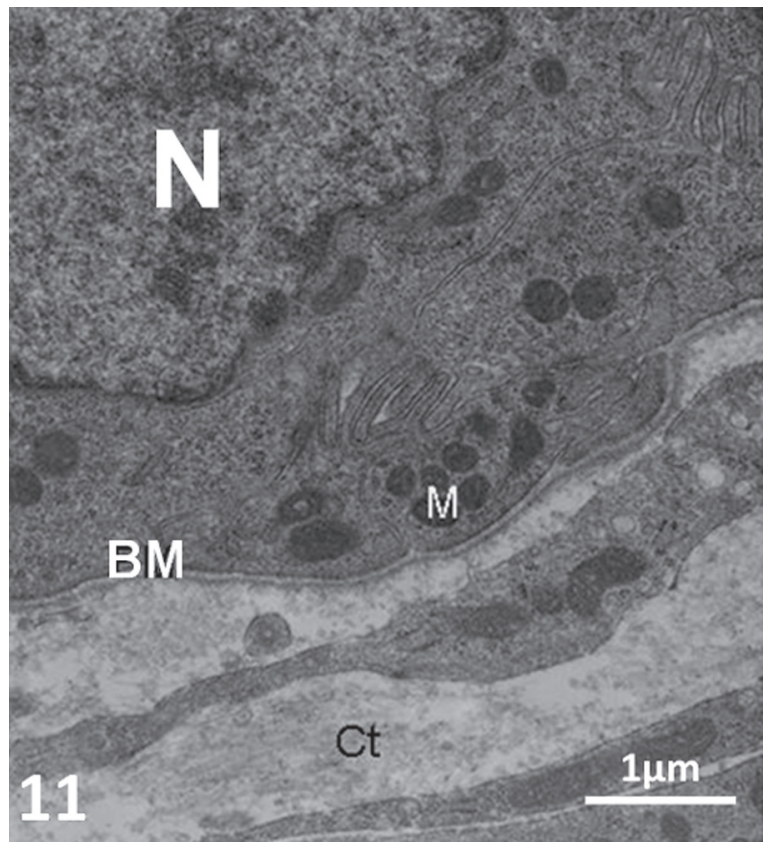

Figure 11. Basal part of secretory gland in endometrial sub-mucosa at mid dioestrus. Notice the slightly indented nucleus (N), variably shaped mitochondria (M) and slightly undulating basement membrane (BM) surrounded by loose connective tissue (Ct). 


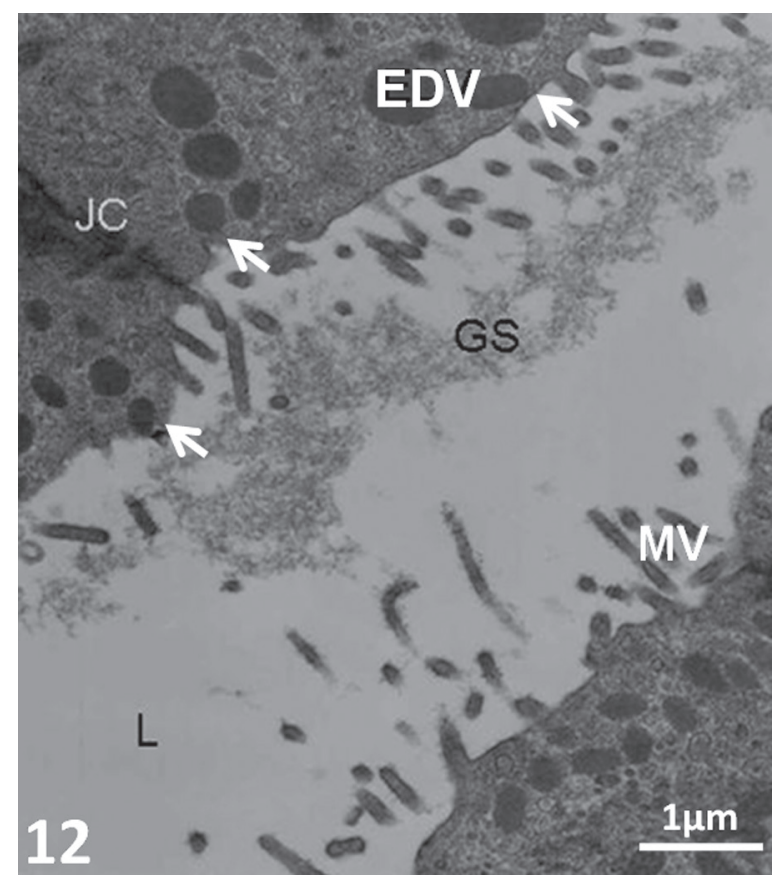

Figure 12. Luminal part of secretory gland in endometrial sub-mucosa at mid dioestrus. Notice the glandular lumen (L) filled with scanty glandular secretion (GS), accumulation of electron dense vesicles (EDV) and microvilli (MV) projection as well as junctional complex (JC) at the apical part of the secretory cells. Also note, exocytosis, fusion of some vesicles to the cell membrane (arrow heads).

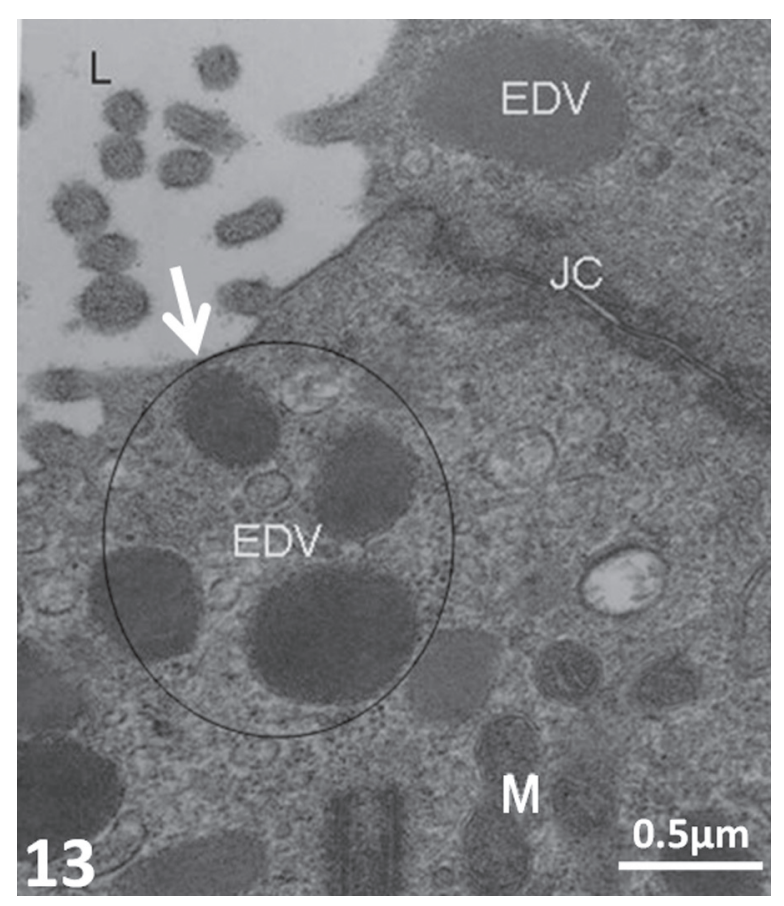

Figure 13. Apex of cell of uterine gland in endometrial sub-mucosa at mid dioestrus. Notice the lumen (L), variably sized electron dense vesicles (EDV), mitochondria (M) and junctional complex (JC) as well as fusion of vesicle to the cell membrane (arrow). uterine ultrastructural features of African giant rat during oestrous cycle and other laboratory animals [4]. Preponderance of spherical mitochondria, organised profiles of parallel Golgi apparatus, numerous electron-dense and electron-lucid vesicles as well as rough endoplasmic reticulum, free ribosomes and polysomes observed at different stages of oestrous cycle suggest a highly secretory uterine mucosa that was supported by the appearance of increasing numbers of secretory vesicles.

The abundance of electron-lucid and electron-dense vesicles at the apex of the cells and their close association with large numbers of mitochondria suggest a high energy requirement [3] and may indicate the transportation of secretory vesicles to their potential site of release. As uterine glands also displayed ultrastructural features similar to the lamina epithelialis, there are convincing and compelling evidences that secretion from these vesicles is probably occurring by a merocrine process. The first evidence is the apparent fusion of the vesicle membrane to the cell membrane suggesting the process of exocytosis. Secondly, the presence of a layer of flocculent materials covering the microvilli indicated that the contents of the vesicles were being expelled at the site. The extremely prominent microvilli would increase the secretory surface area considerably.

Findings that well-developed uterine glands in the lamina propria were observed and some actively secreting uterine glands were lined with ciliated simple columnar, pseudostratified and stratified epithelia also lend credence to earlier report that the weights of both uterine horns as well as the body of uterus increased at mid pro-oestrus and mid oestrus [2]. The uterus passes through three successive phases that correspond to the functional activity of the ovary. The follicular phase, which varies in length, is concurrent with the growth of the follicles and oestrogen secretion. The luteal phase coincides with the functional length of the corpus luteum and the periodic sloughing of the endometrial surface during the oestrous cycle does not cause the extensive bleeding which occurs during the menstruation of primates $[7,13]$. During the follicular phase, the endometrium is conditioned by oestrogen from the ovarian follicles, its vascularity consequently increases and the endometrial glands undergo some growth $[5,6]$. 
In addition, the present study gave insight to the strong immunostaining demonstrated for the oestrogen receptor in the luminal epithelium during pro-oestrus, oestrus and dioestrus [9].

\section{CONCLUSIONS}

In conclusion, the uterine ultrastructure varies during oestrous cycle and uterine glands secretion is by merocrine process. This study complements previous findings obtained in the uterus during oestrous cycle in the Cricetomys. These findings also provide baseline information for future molecular research in the uterus of the African giant rat.

\section{ACKNOWLEDGEMENTS}

The authors thank Prof. J.T. Soley and all the technical staff of the Transmission Electron Microscopy Unit of the Department of Anatomy and Physiology, University of Pretoria for their technical assistance. The study was partly sponsored by the University of Ibadan Senate Research Grant given to Prof. B.O. Oke and the Federal University of Agriculture, Abeokuta, grant given to Dr A.K. Akinloye. The authors are thankful to both institutions.

\section{REFERENCES}

1. Akinloye AK, Abatan MO, Alaka OO, Oke BO (2001) Histo-mophometric and histopathological studies on the effect of calotropis procera (Giant milk weed) on the male reproductive organs of Wistar rats. Afr J Biomed Res, 4: 55-59.

2. Akinloye AK, Oke BO (2009) The uterus of the female African giant rat (Cricetomys gambianus Waterhouse) at
Different stages of the oestrous cycle; gross morphometry. Nigerian Vet J, 30: 1-7.

3. Alberts B, Bray D, Lewis J, Raff M, Roberts K, Watson JD (1989) Energy conversion: mitochondria and chloroplasts. In: Molecular biology of the cell. Garland Publishing, Inc. New York, pp. 341-404.

4. Corbeil LB, Chatterjee A, Foresman L, Westfall JA (1985) Ultrastructure of cyclicchanges in the murine uterus, cervix, and vagina. Tissue Cell, 17: 53-68.

5. Eurell JA, Frappier BL (2006) Female reproductive system. In: Dellmann's textbook of veterinary histology. 6th Ed. Blackwell Publishing, Ames, IA, pp. 256-278.

6. Fawcett DW, Jensh RP (2002) Female reproductive system. In: Bloom and Fawcett's concise histology. 2nd Ed. Arnold Publishers, London, pp. 283-296.

7. Hafez ESE (1987) Reproductive cycles. In: Hafez ESE ed. Reproduction in farm animals. 5th Ed. Lea and Febiger, Philadelphia, pp. 107-129.

8. Knight M (1988) Thermoregulation in the largest African cricetid, the giant rat Cricetomys gambianus. Comparative Bioch Physiol A Physiol, 89: 705-708.

9. Madekurozwa MC, Oke BO, Akinloye AK (2009) Immunohistochemical localization of the progesterone and oestrogen alpha receptors in the uterine horns of the African giant rat (Cricetomys gambianus). Anat Histol Embryol, 38: 419-423.

10. Malekani M, Paulus J (1989) Quelques aspects de la consommation du cricétome, Cricetomys (Rongeurs), par des populations zaïroises. Tropicultura, 7: 141-144.

11. Oke OA, Oke BO (1999) Vaginal cytological changes during the oestrous cycle of the adult female African giant (C. gambianus, Waterhouse). Tropical Vet, 17: 169-180.

12. Skinner JD, Smithers RHN (1990) The mammals of the Southern African subregion. University of Pretoria.

13. Weiss $L$ (1983) The female reproductive system. In: Histology, cell and tissue biology. 5th Ed. Elsevier Science Publishing Co., Inc., New York, pp. 914-943. 